\title{
Aintegumenta and Aintegumenta-Like6 regulate auxin-mediated flower development in Arabidopsis
}

Beth A Krizek

\begin{abstract}
Background: Two related genes encoding AP2/ERF-type transcription factors, AINTEGUMENTA (ANT) and AINTEGUMENTA-LIKE6 (AIL6), are important regulators of floral growth and patterning in Arabidopsis. Evidence suggests that these genes promote several aspects of flower development in response to auxin. To investigate the interplay of ANT, AlL 6 and auxin during floral development, I have examined the phenotypic consequences of disrupting polar auxin transport in ant, ail6 and ant ail6 mutants by either genetic or chemical means.

Results: Plants containing mutations in ANT or AlL6 alone or in both genes together exhibit increased sensitivity to disruptions in polar auxin transport. Both genes promote shoot growth, floral meristem initiation and floral meristem patterning in combination with auxin transport. However, differences in the responses of ant and ail6 single mutants to perturbations in auxin transport suggest that these two genes also have non-overlapping activities in each of these developmental processes.

Conclusions: The enhanced sensitivity of ant and ail6 mutants to alterations in polar auxin transport suggests that these mutants have defects in some aspect of auxin physiology. The inability of ant ail6 double mutants to initiate flowers in backgrounds disrupted for auxin transport confirm the proposed roles for these two genes in floral meristem initiation.
\end{abstract}

\section{Background}

The phytohormone auxin is a central regulator of many aspects of plant growth and development. Within the shoot apical meristem, auxin accumulates in a graded and dynamic manner with sites of auxin maxima correlating with the sites of lateral organ initiation [1-3]. The formation of auxin gradients within the meristem appears to result primarily from directional transport of the hormone and local biosynthesis (reviewed in [4]). Directional transport is mediated by PINFORMED (PIN) proteins, which act as auxin efflux carriers and exhibit polarized plasma membrane localization [5]. Mutations in PIN1 result in inflorescences that do not initiate floral meristems and instead grow as pin-like structures [6]. This phenotype can be rescued by application of auxin to the meristem, demonstrating the importance of auxin to floral meristem initiation and

Correspondence: krizek@sc.edu

Dept. of Biological Sciences, University of South Carolina, Columbia, SC, 29208, USA the pivotal role that PIN1 plays in the generation of auxin gradients within the meristem [7]. These conclusions are also supported by studies showing that wildtype plants grown in the presence of the auxin transport inhibitor N-1-naphthylphthalamic acid (NPA) phenocopy pin1 mutants [6].

In addition to regulating floral meristem outgrowth, auxin also regulates floral organ development. Disruptions in polar auxin transport via transient NPA application result in flowers with reduced numbers of floral organs, narrower sepals and petals, malformed anthers and gynoecium with altered patterning [8]. These alterations in gynoecium patterning are very similar to those resulting from mutations in ETTIN (ETT), which encodes an auxin response factor (ARF) [8-10]. Mutations in PIN1 or PINOID (PID), which encodes a Ser-Thr kinase that controls PIN1 polarity, can produce several abnormal flowers prior to inflorescence termination in a pin-like structure [6,11-13]. These flowers exhibit a range of defects that can include alterations in organ number
C Biomed Central

C 2011 Krizek et al; licensee BioMed Central Ltd. This is an open access article distributed under the terms of the Creative Commons Attribution License (http://creativecommons.org/licenses/by/2.0), which permits unrestricted use, distribution, and reproduction in any medium, provided the original work is properly cited. 
(typically fewer sepals and stamens and more petals), fusion of floral organs, and valveless gynoecia [6,13]. Disruptions in flower development have also been observed in plants defective in auxin biosynthesis. Biosynthesis of the major auxin, indole-3-acetic acid (IAA), involves both tryptophan (Trp)-dependent and Trp-independent pathways (reviewed in [14]). Mutations in multiple members of the YUCCA $(Y U C)$ family of flavin monooxygenases, which act in the tryptamine (TAM) Trp-dependent pathway, display reductions in floral organ number, altered organ morphology, valveless gynoecia and sterility [15]. Similar phenotypes are observed in plants mutant for tryptophan aminotransferase TAA1 and the related TAR2 gene, which act in the indole-3-pyruvic acid (IPA) Trp-dependent pathway $[16,17]$.

The molecular mechanisms by which auxin regulates floral meristem outgrowth and organogenesis within the flower are not well defined. Real time imaging of inflorescence meristems has demonstrated that PIN1 upregulation in floral anlagen is correlated with downregulation of the meristem regulator SHOOTMERISTEMLESS (STM) as well as the boundary protein CUPSHAPED COTYLEDON 2 (CUC2) [2]. Two genes that are likely to promote floral meristem initiation and specification downstream of auxin are the growth-promoting gene $A N T$ and the floral meristem identity gene $L E A F Y(L F Y)$. These two genes are early markers of a floral meristem fate and their expression is altered in pin1 mutants [18-20]. In addition, exposure of wild-type inflorescences to NPA results in a decrease in $A N T$ mRNA accumulation in floral anlagen as early as 24 hours after treatment [21]. These data are consistent with a model in which auxin accumulation upregulates $A N T$ and $L F Y$ expression to promote primordium outgrowth and floral identity, respectively [18]. However ant mutants do not display any defects in floral meristem initiation. Thus either ANT does not play a role in floral meristem initiation or it acts in parallel with other genes to promote primordium outgrowth.

Besides its proposed role in floral meristem initiation, $A N T$ regulates floral meristem patterning, specification of floral organ identity, growth of floral organs and gynoecium patterning [19,21-25]. In two of these processes (lateral organ growth and gynoecium patterning) $A N T$ function been linked directly to auxin $[25,26]$. $A N T$ promotes growth downstream of the auxin-inducible gene $A R G O S$ (auxin-regulated gene involved in organ size), while in maturing organs $A N T$ expression is repressed by $A R F 2$, a repressor of organ growth [26,27]. In the gynoecium, ANT acts together with REVOLUTA and polar auxin transport to specify development of the carpel medial domain [25].

In several of its roles in flower development, ANT acts in a partially redundant manner with the related
AINTEGUMENTA-LIKE6 (AIL6). While ail6 flowers have a wild-type appearance, ant ail6 flowers have more severe defects than ant. ant ail6 flowers lack petals, stamens and normal gynoecium, are dramatically reduced in size, and exhibit defects in floral organ positioning [21]. Altered expression of the auxin-responsive reporter AGH3-2:GUS in ant ail6 inflorescence meristems and flowers suggests that these floral defects may be a consequence of altered patterns of auxin accumulation and/ or responsiveness [21]. In addition to floral defects, ant ail6 plants exhibit decreased apical dominance, reduced stature, and altered vascular patterning, phenotypes similar to those found in plants disrupted in auxin physiology [21].

Like ANT, AIL6 expression is upregulated in incipient lateral organ primordia. This suggests that AIL6 might act redundantly with $A N T$ in floral meristem initiation, but ant ail6 double mutants are still able to initiate floral meristems. ant ail6 inflorescence meristems do eventually stop initiating flowers, but this is due to a general growth arrest of the entire inflorescence apex [21] rather than specific termination of floral meristem initiation with continued growth of the inflorescence apex as is observed in pin1 mutants. Thus, it is still not known whether $A N T$ and AIL6 regulate floral meristem initiation.

To further probe the roles of ANT and AIL6 in floral development processes potentially regulated by auxin, I have examined the consequences of losing ANT and AIL6 function singly or together in plants compromised for auxin transport via genetic or pharmacological means. These experiments demonstrate that both mutants are sensitized to defects in auxin transport, consistent with a role for these genes in regulation of some aspect of auxin physiology during flower development. The phenotypic consequences of loss of $A N T$ function and loss of AIL6 function under the same conditions are somewhat different, suggesting that these two genes make distinct contributions to early stages of flower development. Floral meristem initiation is completely suppressed in plants compromised in polar auxin transport and containing mutations in both $A N T$ and AIL6, demonstrating that these two genes do function in the initiation of floral meristems from the inflorescence meristem.

\section{Findings}

Mutations in ANT and AIL6 enhance the floral initiation and floral patterning defects of pid mutants

Because pid mutants make several flowers prior to termination of the inflorescence meristem, they provide a sensitized background in which to study the role of $A N T$ and AIL6 in auxin-mediated floral meristem initiation. I introduced ant-4, ail6-2 and ant-4 ail6-2 into the 
strong pid-1 allele and the intermediate pid-2 allele [13]. No flowers were produced in pid-1 ant-4 ail6-2 or pid-2 ant-4 ail6-2 triple mutants (Figure 1A, B). While flowers were produced in pid-1 ant-4, pid-1 ail6-2, pid-2 ant-4 and pid-2 ail6-2 plants, they were reduced in number compared to pid-1 and pid-2 single mutants (Figure 1A, B). These results indicate that ANT and AIL6 have partially overlapping roles in promoting floral meristem initiation.

The flowers produced by pid-1 ant-4 and pid-1 ail6-2 plants exhibited more severe defects than those observed in pid-1 (Figure 2A-F). pid-1 ant-4 flowers were extremely small with reduced numbers of petals and stamens (Figure 2C, D). A similar phenotype was observed in pid-2 ant-4 flowers (Figure 2G, H). The dramatic effect on petal number was surprising since ant-4 flowers exhibit only slight reductions in petal number in early-arising flowers while pid flowers have increased numbers of petals [13,21]. pid-1 ail6-2 flowers resembled pid-1 flowers except for an increased incidence of sepal fusion. Eighty percent of pid-1 ail6-2 flowers exhibited sepal fusion while $41.2 \%$ of pid-1 flowers exhibited sepal fusion (Figure 2E, F). ail6-2 single mutants exhibited no sepal fusion or any other alterations in flower development compared with wild type [21].

\section{Mutations in ANT and AIL6 enhance shoot defects in plants genetically disrupted for auxin transport}

The pid-1 ant-4 ail6-2 triple mutants also exhibited severe defects in leaf size and stem growth. Leaves of the triple mutant were thinner and shorter than in pid-1 (Figure 1D). The naked pin-like inflorescences of these plants were extremely short in height and often not visible above the basal rosette of the plant (Figure 1C, D). Leaf size and plant height were partially compromised in pid-1 ant-4 plants but not significantly altered in pid1 ail6-2 plants (Figure 1C, D). Similar results were obtained with the pid-2 allele.

I also investigated the effect on introducing ant-4 ail62 into pin mutant backgrounds using pin1-1 (strong allele in Col) and pin1-3 (strong allele in Ler). Similar to the results with pid mutants, pin1-3 ant-3 ail6-2 plants produce small leaves (Figure 1E) and short inflorescences (Figure 1F). Inflorescence height was somewhat variable in these plants (Figure $1 \mathrm{~F}$ ); in most cases the pin-like inflorescence did not extend beyond the basal rosette. Similar results were obtained with the pin1-1 allele. Interestingly, pin1-3 ant-4 and pin1-3 ail6-2 double mutants exhibited distinct phenotypes. pin1-3 ant-4. plants exhibited enhanced branching compared to pin13, while pin1-3 ail6-2 plants only rarely produced cauline leaves or axillary branches from the primary inflorescence (Figure 1G). ant mutants exhibit increased sensitivity to the effects of NPA on floral organ development

I next examined the consequence of inhibiting polar auxin treatment via application of $10 \mu \mathrm{M}$ NPA to ant-4 flowers. This concentration of NPA had some phenotypic consequences on wild-type Arabidopsis flower development, such as reductions in stamen number (Figure 3G) but does not dramatically reduce floral meristem initiation, as is the case when wild-type inflorescences are treated with $100 \mu \mathrm{M}$ NPA [8]. Thus it can be used to examine the relative sensitivity of ant- 4 and Ler flowers to NPA treatment. Examination of mock and NPA-treated ant-4 plants revealed temporally distinct phenotypes. The first effects of NPA treatment were observed in ant-4 flowers opening approximately 10 days after treatment and continued through day 12 after treatment. A second phenotypic class was observed in ant-4 flowers opening 13-15 days post treatment.

In 10-12 day post NPA-treated ant-4 flowers, second whorl petals were often replaced with filaments or very thin petals (Figure 3A-C). Organs were classified as filaments if they were radially symmetric and as thin petals if they had a flattened appearance. Alterations in petal development were not observed in 10-12 day post mock-treated ant-4 plants or NPA-treated Ler plants (Figure 3A, C). To further characterize the second whorl organs that develop in these NPA-treated ant-4 flowers, SEM was used to examine their epidermal surface morphologies. Epidermal cells of the filaments lacked petal cell shape and cuticular thickenings suggesting that these organs did not possess any petal identity (Figure 3D). Epidermal cells of thin petals did show cuticular thickenings characteristic of petals (Figure 3E). However, these organs often lacked the conical cells normally present on the adaxial surface of petals, suggesting that these organs, like the filaments, had disruptions in organ polarity. No significant differences were observed in the numbers of sepals, stamens or carpels of 10-12 day post NPA-treated ant-4 or Ler plants (Figure 3F). Occasionally, a valveless gynoecia phenotype was observed in the fourth whorl of 10-12 day post NPA-treated ant-4 flowers.

In 13-15 day post NPA-treated ant-4 plants, petals were often missing entirely (rather than being replaced with filaments) (Figure 3G) and sepals were often fused to each other (Figure 3H-J). The incidence of sepal fusion was much greater in NPA-treated ant-4 flowers (0.90 incidence/flower) compared with NPA-treated Ler flowers (0.20 incidence/flower) (Figure $3 \mathrm{H}$ ).

The primary effect of $10 \mu \mathrm{M}$ NPA treatment on Ler flowers was a decrease in stamen number (Figure $3 G$ ). Stamen number after NPA treatment was similar in Ler and ant-4 flowers even though mock or untreated ant-4. 

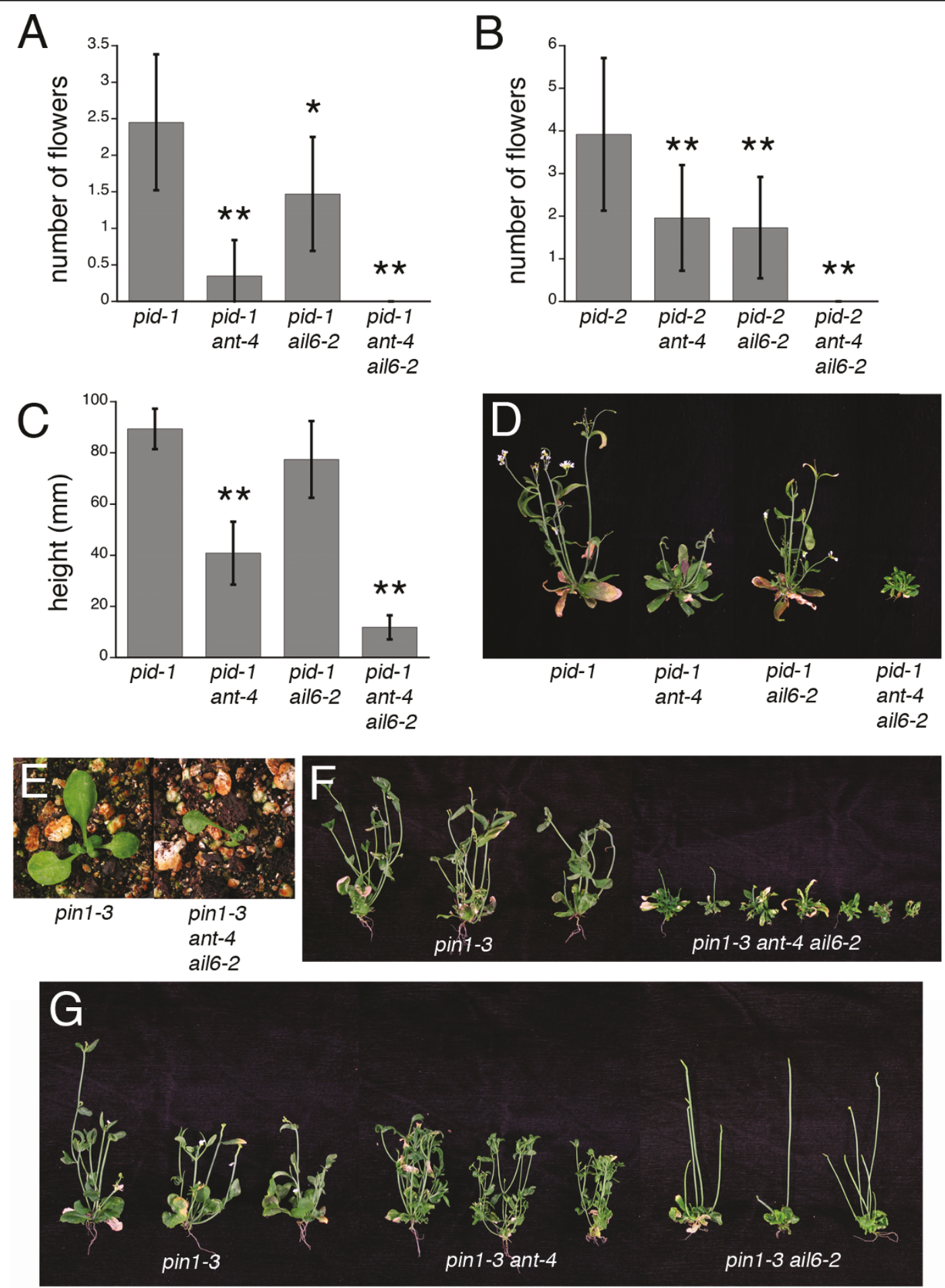

Figure 1 ant- 4 and ail6-2 enhance the shoot and inflorescence defects of pid and pin 1 mutants. A. Number of flowers produced on pid-1, pid-1 ant-4, pid-1 ail6-2 and pid-1 ant-4 ail6-2 plants. B. Number of flowers produced on pid-2, pid-2 ant-4, pid-2 ail6-2 and pid-2 ant-4 ail6-2 plants. C. Height of pid-1, pid-1 ant-4, pid-1 ail6-2 and pid-1 ant-4 ail6-2 plants. Data in A-C are means \pm SD. P values were determined by unpaired Student's t test (* $\left.P \leq 0.01,{ }^{* *} P \leq 0.0001\right)$. D. 39 day old pid-1, pid-1 ant-4, pid-1 ail6-2 and pid-1 ant-4 ail6-2 plants. E. 23 day old pin1-3 and pin 1-3 ant-4 ail6-2 plants. F. 54 day old pin 1-3 and pin 1-3 ant-4 ail6-2 plants. G. 54 day old pin 1-3, pin 1-3 ant-4 and pin 1-3 ail6-2 plants. 

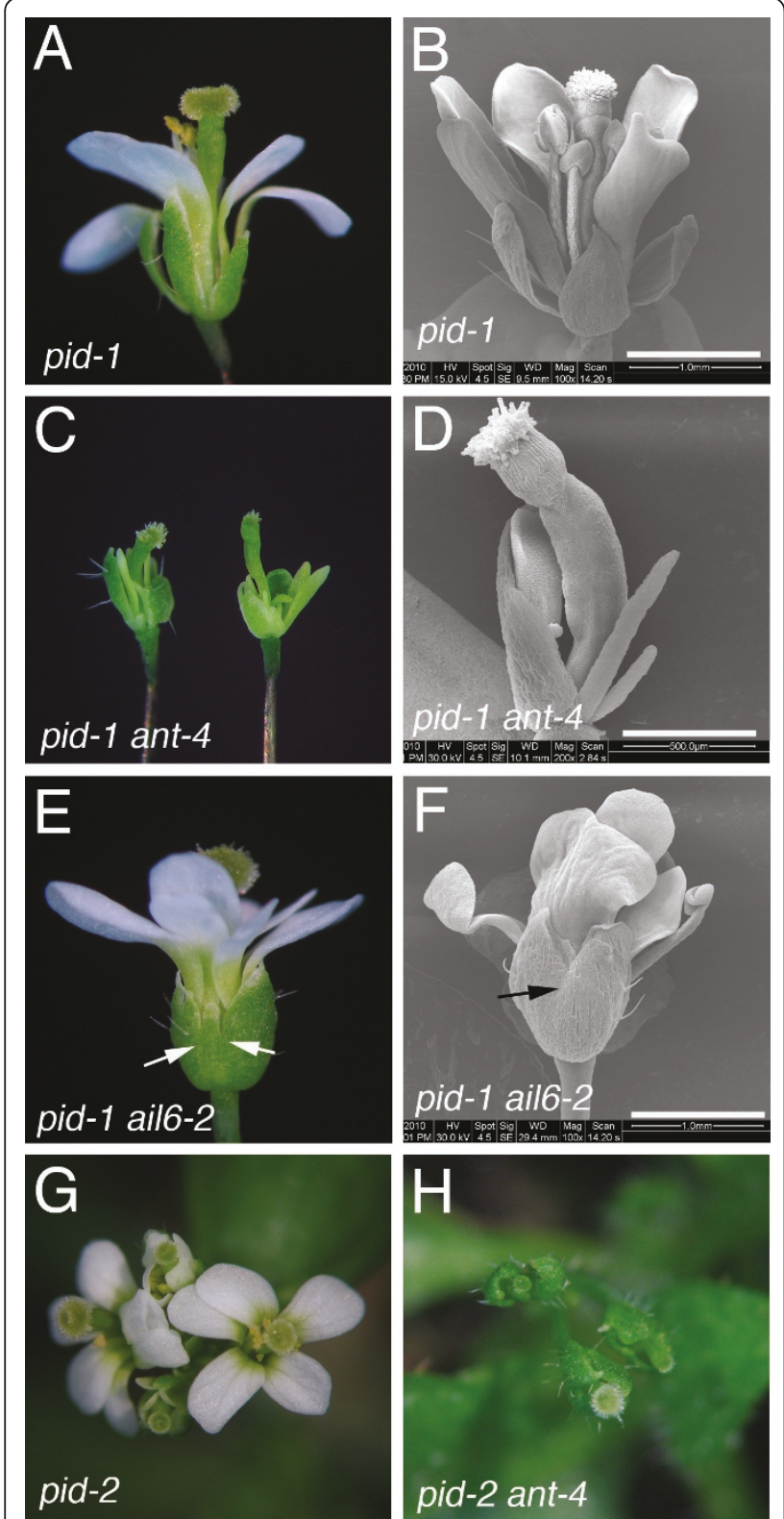

Figure 2 ant-4 and ail6-2 enhance the floral defects of pid. A. pid-1 flower. B. Scanning electron micrograph of a pid-1 flower. C. pid-1 ant-4 flowers. D. Scanning electron micrograph of a pid-1ant4 flower. E. pid-1 ail6-2 flower. Arrows point to fused sepals. F. Scanning electron micrograph of a pid-1 ail6-2 flower. The arrow points to a region of sepal fusion. G. pid-2 inflorescence. H. pid-2 ant-4 inflorescence. Size bars correspond to $1 \mathrm{~mm}$ in $B$, F; $500 \mu \mathrm{M}$ in $\mathrm{D}$

flowers produced fewer stamens than Ler (Figure 3G). In addition, the incidence of stamen fusion was increased in 10-15 day post NPA-treated ant-4 flowers (0.59 incidence/flower) compared to NPA-treated Ler flowers (0.07 incidence/flower) (Figure $3 \mathrm{H}$ ). ail6 mutants exhibit increased sensitivity to the effects of NPA on floral meristem initiation

Treatment of Ler inflorescences with $10 \mu \mathrm{M}$ NPA had no dramatic effects on floral meristem initiation (Figure 4A, B). However, treatment of ail6-2 inflorescences with $10 \mu \mathrm{M}$ NPA resulted in a temporary suspension of floral meristem initiation (Figure 4C, D). Since primordia initiation was restarted at a later date, there was a gap in the developmental series of floral buds represented on an inflorescence (Figure 4C, D). SEM analyses suggested that this time period corresponds to the initiation of at least six flowers in wild type (Figure 4B, D). Floral organ number was similarly affected in NPA-treated Ler and ail6-2 flowers (Figure 4E). While sepal number was similar in NPA-treated Ler and ail6-2 flowers, the incidence of sepal fusion was increased in NPA-treated ail6-2 flowers (0.46 incidence/flower) compared to NPA-treated Ler flowers (0.16 incidence/flower) (Figure 4F, G). No obvious effect on floral meristem initiation or floral organ development was observed in ant-4 ail6-2 plants treated with NPA as compared with mocktreated plants. Any effect on floral meristem initiation would be difficult to observe since termination of the inflorescence meristem occurs in untreated ant-4 ail6-2 inflorescences prior to the expected effect of NPA treatment.

\section{Discussion}

\section{ANT and AIL6 promote floral meristem initiation}

Although it has been proposed that ANT promotes primordium outgrowth in response to auxin accumulation, neither ant nor ant ail6 double mutants display defects in floral meristem initiation from the inflorescence meristem. The results presented here provide the first evidence that ANT and AIL6 are important factors regulating floral meristem initiation. Combining either ant or ail6 with pid reduces the number of flowers initiated by the inflorescence meristem, while the loss of both ANT and AIL6 function together prevents the formation of any flowers in pid mutants. These results suggest that ANT and AIL6 act in a redundant fashion to promote floral meristem initiation. Other pieces of evidence however suggest that the activities of these two genes are not completely overlapping and that AIL6 may play a more important role in this process as compared with $A N T$. Floral meristem initiation was temporarily terminated in ail62 inflorescences treated with $10 \mu \mathrm{M}$ NPA but not in Ler or ant-4 inflorescences similarly treated. Thus floral meristem initiation is inhibited by lower levels of NPA in ail6-2 inflorescences. Furthermore, loss of AIL6 function in the pin1-3 background results in a nearly complete loss of lateral branches from inflorescences. 

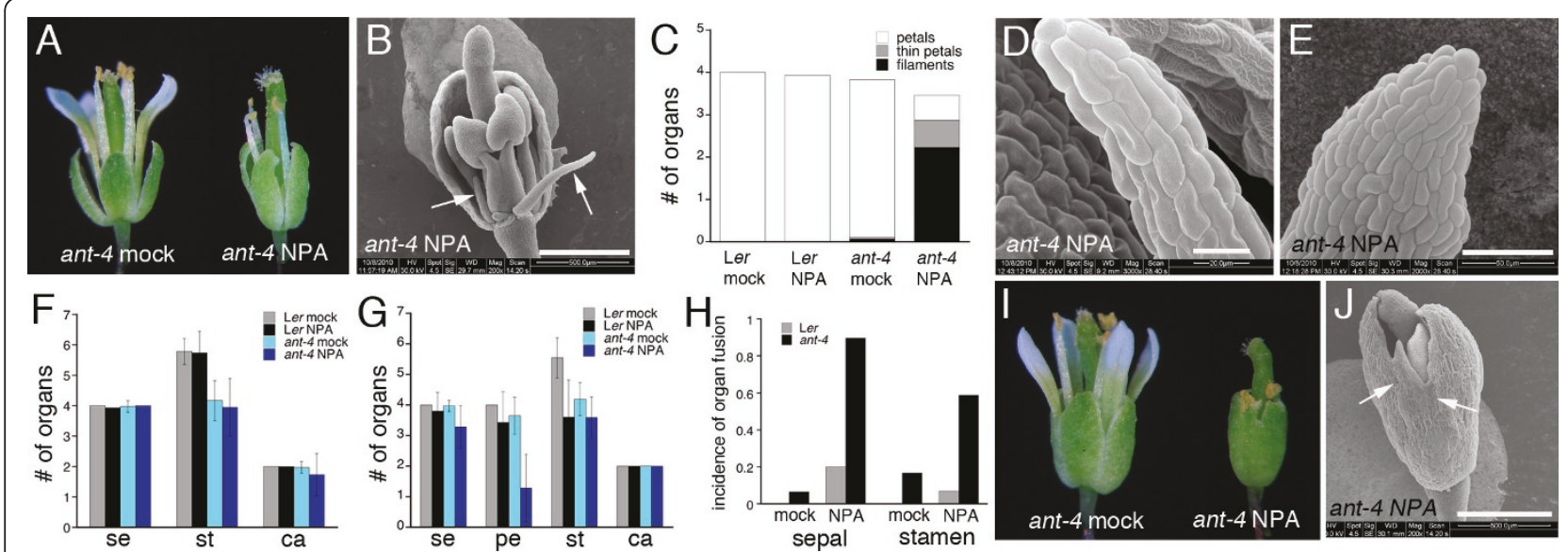

Figure 3 Effects of NPA treatment on ant-4 flowers. A. Mock (left) and NPA (right) treated ant-4 flowers 11 days post treatment. B. Scanning electron micrograph of a NPA treated ant-4 flower fixed 9 days post treatment. Arrows point to filaments present in the second whorl. C. Organ types found in second whorl of mock and NPA-treated Ler and ant-4 flowers. D. Scanning electron micrograph of a second whorl filament. E. Scanning electron micrograph of a second whorl thin petal. F. Floral organ number in mock and NPA treated Ler and ant-4 inflorescences from 10-12 days post treatment. Data are means \pm SD. G. Floral organ number in mock and NPA treated Ler and ant-4 inflorescences from 13-15 days post treatment. Data are means \pm SD. H. Incidence of sepal fusion and stamen fusion in mock and NPA treated Ler and ant-4 inflorescences. Sepal fusion was counted in flowers maturing at 13-15 days post treatment while stamen fusion was counted in flowers maturing 10-15 days post treatment. I. Mock (left) and NPA (right) treated ant-4 flowers 13 days post treatment. J. Scanning electron micrograph of an NPA-treated ant-4 flower that exhibits sepal fusion. Arrows point to fused sepals. Size bars correspond to $500 \mu \mathrm{M}$ in $\mathrm{B}, \mathrm{J} ; 20 \mu \mathrm{M}$ in $\mathrm{D} ; 50 \mu \mathrm{M}$ in $\mathrm{E}$.

\section{ANT and AIL6 act with polar auxin transport in floral meristem patterning}

One defect in flower development observed in mutants with disruptions in polar auxin transport such as pid [13] or in wild-type plants treated with NPA was fusion of floral organs. This may be a consequence of alterations in the positioning or outgrowth of organ primordia from the floral meristem and/or the inability to establish boundaries between adjacent organs. It is known that polar auxin transport is critical to cotyledon boundary formation during embryogenesis [28]. In postembryonic development, separation between lateral organs and the meristems from which they arise is also likely to involve polar auxin transport (reviewed in [4,29]). Live imaging of inflorescence meristem shows that upregulation of PIN1 expression in lateral organ founder cells was correlated with reduced expression of meristem and boundary markers such as STM and CUC2 [2]. Correspondingly, boundary genes such as JAGGED LATERAL ORGAN (JLO) appear to repress PIN1 and promote STM expression in boundary cells [30]. These antagonistic relationships result in the separation of indeterminate cells within the meristem and determinate cells within the lateral organ primordia [29]. The results presented here showing floral organ fusion in some NPA treated wildtype flowers suggest that a common mechanisms is involved in organ separation in shoot apical meristems and floral meristems. Sepal fusion was enhanced in pid-1 ail6-2 flowers compared to pid-1 flowers and in NPA treated ant-4 and ail6-2 flowers as compared with NPA treated Ler flowers. Thus, ANT and AIL6 appear to function together with polar auxin transport in separation of sepals, while ANT also acts in stamen boundary specification. These data provide a link between auxin and the role of ANT and AIL6 in floral meristem patterning.

\section{ANT promotes petal development in combination with auxin transport}

ant-4 mutants in which polar auxin transport was reduced also exhibited severe effects on petal development. Few petals were initiated in pid ant-4 double mutants or in the later-arising NPA-treated ant-4 flowers. The distinct temporal effects observed after NPA treatment on ant-4 flowers were likely a consequence of the floral development stage at which auxin transport was inhibited. Flowers maturing 10-12 days after NPA treatment presumably correspond to those in which sepal and petal primordia were already initiated while flowers maturing 13-15 days after NPA treatment most likely correspond to younger floral meristems in which sepal and petal primordia had not yet initiated. In Ler plants, the length of time between floral meristem initiation and flower opening is approximately 13.25 days [31]. In the earliest flowers affected by NPA treatment, second whorl organs were present but often developed as filaments or very thin petals. The lack of petal blade outgrowth and the absence of conical cells in thin petals indicate that petal polarity is disrupted. A role for auxin 


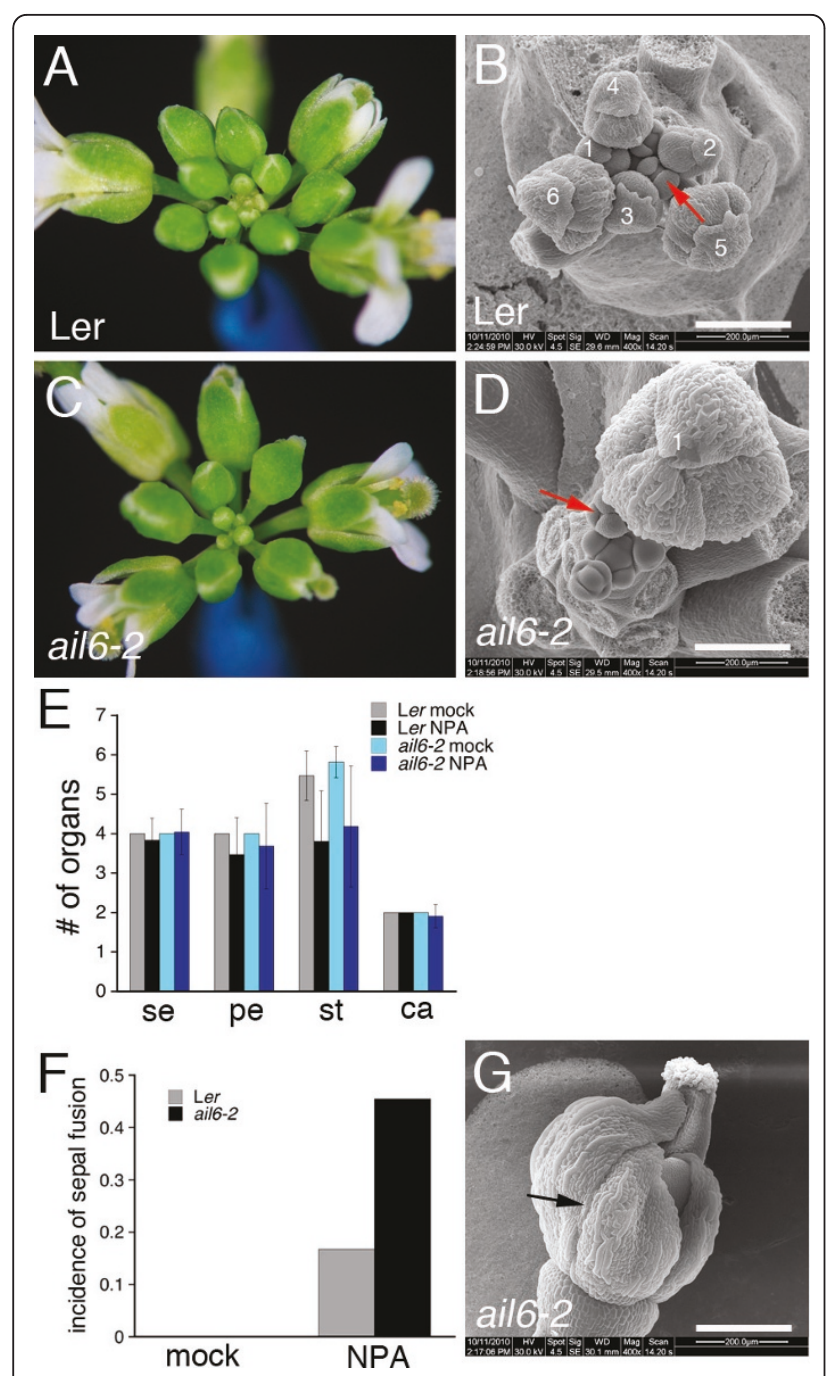

Figure 4 Effects of NPA treatment on ail6-2 inflorescences. A. Ler inflorescence 11 days post NPA treatment. B. Scanning electron micrograph of Ler inflorescence fixed 9 days post NPA treatment. The red arrow points to a stage 4 flower and the numbers mark all flowers initiated prior to the stage 4 flower. C. ail6-2 inflorescence 11 days post NPA treatment. D. Scanning electron micrograph of ail6-2 inflorescence fixed 9 days post NPA treatment. The red arrow points to a stage 4 flower. The number 1 marks the last flower initiated prior to temporary termination of floral meristem initiation. E. Floral organ number in mock and NPA treated Ler and ail6-2 inflorescences from 12-15 days post treatment. Data are means \pm SD. F. Incidence of sepal fusion in mock and NPA treated Ler and ail6-2 flowers counted 12-15 days post treatment. G. Scanning electron micrograph of an NPA-treated ail6-2 flower that exhibits sepal fusion. Arrows point to a region of fusion. Size bars correspond to $200 \mu \mathrm{M}$ in $\mathrm{B}, \mathrm{D} ; 200 \mu \mathrm{M}$ in $\mathrm{G}$.

in mediating adaxial/abaxial patterning during organogenesis has been suggested by the phenotype of plants lacking two related ARFs, ETT and ARF4 [32]. ett arf4 double mutants have defects in lateral organ polarity that mimic loss of function mutations in KANADI genes that specify abaxial identity [32]. In later-arising NPA- treated ant-4 flowers, second whorl organs were usually missing suggesting that polar auxin transport is required for petal initiation. It is not clear why petal initiation is particularly sensitive to NPA treatment in the ant-4 background.

\section{Conclusions}

Here I show that combining mutations in $A N T$ and AIL6 with disruptions in polar auxin transport results in severe effects on shoot and flower development. ANT and AIL6 appear to have both overlapping and distinct roles in the process of floral meristem initiation downstream of auxin, while $A N T$ appears to play a more important role than AIL6 in later stages of auxinmediated floral meristem patterning and floral organogenesis. The results are consistent with a model in which ant and ail6 mutants are disrupted in some aspect of auxin physiology. Furthermore they support the idea that all of the different functions of these two genes are linked with auxin dynamics within shoot tissues.

\section{Methods}

\section{Plant materials and growth conditions}

Arabidopsis thaliana ecotype Landsberg erecta (Ler) was used as the wild type. Other mutants used in the study were pid-1 [13], pid-2 [13], pin1-1 [6], pin1-3 [13], ant-4 [33] and ant-4 ail6-2 [21]. Plants were grown on a soil mixture of Metro-Mix 360:perlite:vermiculite (5:1:1) under continuous light or in 16 hour days (100-150 $\mu \mathrm{mol} / \mathrm{m}^{2} / \mathrm{s}$ ) at a temperature of $22^{\circ} \mathrm{C}$.

\section{Genetics and PCR genotyping}

ant -4 and ant $-4 /+$ ail6-2 plants were crossed to pid $-1 /+$, pid-2, pin1-1/+ and pin1-3/+ plants. Double and triple mutants were identified in the F2 or later generations as plants with new phenotypes and confirmed by PCR genotyping. ant-4 and ail6-2 were PCR genotyped as described previously [21]. pin1-3 was PCR genotyped using PIN1-5: 5'-caccgctacgaacgatcatcaa-3' and PIN1-6: 5'-atgctttctgctgtgaagccag-3'. Digestion of the PCR product with ScaI produced two fragments of $449 \mathrm{bp}$ and $529 \mathrm{bp}$ for wild type and an intact $978 \mathrm{bp}$ fragment for pin1-3.

\section{SEM}

Tissue for SEM was fixed, dried, dissected and coated as described previously [23]. SEM analyses were performed on a FEI Quanta 200 ESEM.

\section{NPA treatment}

Ler, ant-4, ail6-2 and ant-4 ail6-2 inflorescences were painted with a $10 \mu \mathrm{M}$ NPA (in 0.1\% DMSO, $0.01 \%$ 
Silwet L-77) solution or a mock (0.1\% DMSO, 0.01\% Silwet L-77) solution twice (at time zero and 7 hours).

\section{Acknowledgements and Funding}

I thank Mitsuhiro Aida for pin 1-3 seeds and genotyping information, the ABRC for pid-1, pid-2 and pin1-1 seeds, and Soumitra Ghoshroy and the Electron Microscopy Center staff for advice on the use of the SEM. This work was supported by National Science Foundation (NSF) grant IOS 0922367.

Authors' contributions

BAK is solely responsible for this work.

\section{Competing interests}

The author declares that they have no competing interests.

Received: 21 December 2010 Accepted: 7 June 2011

Published: 7 June 2011

\section{References}

1. Benkova E, Michniewicz M, Sauer M, Teichmann T, Seifertova D, Jurgens G, Friml J: Local, efflux-dependent auxin gradients as a common module for plant organ formation. Cell 2003, 115:591-602.

2. Heisler MG, Ohno C, Das P, Sieber P, Reddy GV, Long JA, Meyerowitz EM: Patterns of auxin transport and gene expression during primodium development revealed by live imaging of the Arabidopsis inflorescence meristem. Curr Biol 2005, 15:1899-1911.

3. Reinhardt D, Eva-Rachele P, Stieger P, Mandel T, Baltensperger K, Bennett M, Traas J, Friml J, Kuhlemeier C: Regulation of phyllotaxis by polar auxin transport. Nature 2003, 426:255-260

4. Vernoux T, Besnard F, Traas J: Auxin at the shoot apical meristem. Cold Spring Harb Perspect Biol 2010, 2:a001487.

5. Galweiler L, Guan C, Muller A, Wisman E, Mendgen K, Yephremov A Palme K: Regulation of polar auxin transport by AtPIN1 in Arabidopsis vascular tissue. Science 1998, 282:2226-2230.

6. Okada K, Ueda J, Komaki MK, Bell CJ, Shimura Y: Requirement of the auxin polar transport system in early stages of Arabidopsis floral bud formation. Plant Cell 1991, 3:677-684.

7. Reinhardt D, Mandel T, Kuhlemeier C: Auxin regulates the intiation and radial position of plant lateral organs. Plant Cell 2000, 12:507-518.

8. Nemhauser JL, Feldman $L$, Zambryski PC: Auxin and ETTIN in Arabidopsis gynoecium morphogenesis. Development 2000, 127:3877-3888.

9. Sessions A, Nemhauser JL, McColl A, Roe JL, Feldman KA, Zambryski PC: ETTIN patterns the Arabidopsis floral meristem and reproductive organs. Development 1995, 124:4481-4491.

10. Sessions RA, Zambryski PC: Arabidopsis gynoecium structure in the wild type and in ettin mutants. Development 1995, 121:1519-1532.

11. Christensen SK, Dagenais N, Chory J, Weigel D: Regulation of auxin response by the protein kinase PINOID. Cell 2000, 100:469-478.

12. Friml J, Yang $X$, Michniewicz M, Weijers D, Quint A, Tietz O, Benjamins $R$, Ouwerkerk PB, Ljung K, Sandberg G, et al: A PINOID-dependent binary switch in apical-basal PIN polar targeting direct auxin efflux. Science 2004, 306:862-865

13. Bennett SRM, Alvarez J, Bossinger G, Smyth DR: Morphogenesis in pinoid mutants of Arabidopsis thaliana. Plant J 1995, 8:505-520.

14. Woodward AW, Bartel B: Auxin: Regulation, Action and Interaction. Ann Bot 2005, 95:707-735.

15. Cheng Y, Dai X, Zhao Y: Auxin biosynthesis by the YUCCA flavin monooxygenase controls the formation of floral organs and vascular tissues in Arabidopsis. Gene Dev 2006, 20:1790-1799.

16. Stepanova AN, Robertson-Hoyt J, Yun J, Benavente LM, Xie D-Y, Dolezal K, Schlereth A, Jurgens G, Alonso JM: TAA1-mediated auxin biosynthesis is essential for hormone crosstalk and plant development. Cell 2008, 133:177-191.

17. Tao Y, Ferrer J-L, Ljung K, Pojer F, Hong F, Long JA, Li L, Morenos JE, Bowman ME, Ivans $L$, et al: Rapid synthesis of auxin via a new tryptophan-dependent pathway is required for shade avoidance in plants. Cell 2008, 133:164+176.

18. Vernoux T, Kronenberger J, Grandjean O, Laufs P, Traas J: PIN-FORMED 1 regulates cell fate at the periphery of the shoot apical meristem. Development 2000, 127:5157-5165.
19. Elliott RC, Betzner AS, Huttner E, Oakes MP, Tucker WQJ, Gerentes D, Perez P, Smyth DR: AINTEGUMENTA, an APETALA2-like gene of Arabidopsis with pleiotropic roles in ovule development and floral organ growth. Plant Cell 1996, 8:155-168.

20. Weigel D, Alvarez J, Smyth DR, Yanofsky MF, Meyerowitz EM: LEAFY controls floral meristem identity in Arabidopsis. Cell 1992, 69:843-859.

21. Krizek BA: AINTEGUMENTA and AINTEGUMENTA-LIKE6 act redundantly to regulate Arabidopsis floral growth and patterning. Plant Physiol 2009, 150:1916-1929.

22. Klucher KM, Chow H, Reiser L, Fischer RL: The AINTEGUMENTA gene of Arabidopsis required for ovule and female gametophyte development is related to the floral homeotic gene APETALA2. Plant Cell 1996, 8:137-153.

23. Krizek BA: Ectopic expression of AINTEGUMENTA in Arabidopsis plants results in increased growth of floral organs. Dev Genet 1999, 25:224-236.

24. Mizukami Y, Fischer RL: Plant organ size control: AINTEGUMENTA regulates growth and cell numbers during organogenesis. Proc Natl Acad Sci USA 2000, 97:942-947.

25. Nole-Wilson S, Azhakanandam S, Franks RG: Polar auxin transport together with AINTEGUMENTA and REVOLUTA coordinate early Arabidopsis gynoecium development. Dev Biol 2010, 346:181-195.

26. Hu Y, Xie A, Chua N-H: The Arabidopsis auxin-inducible gene ARGOS controls lateral organ size. Plant Cell 2003, 15:1951-1961.

27. Schruff MC, Spielman M, Tiwari S, Adams S, Fenby N, Scott RJ: The AUXIN RESPONSE FACTOR 2 gene of Arabidopsis links auxin signaling, cell division, and the size of seeds and other organs. Development 2005, 133:251-261

28. Furutani M, Vernoux T, Traas J, Kato T, Tasaka M, Aida M: PIN-FORMED and PINOID regulate boundary formation and cotyledon developmetn in Arabidopsis embryogenesis. Development 2004, 131:5021-5030.

29. Rast MI, Simon R: The meristem-to-organ boundary: more than an extremity of anything. Curr Opin Genet Dev 2008, 18:287-294.

30. Borghi $L$, Bureau M, Simon R: Arabidopsis JAGGED LATERAL ORGANS is expressed in boundaries and coordinates KNOX and PIN activity. Plant Cell 2007, 19:1795-1808.

31. Smyth DR, Bowman JL, Meyerowitz EM: Early flower development in Arabidopsis. Plant Cell 1990, 2:755-767.

32. Pekker I, Alvarez JP, Eshed Y: Auxin response factors mediate Arabidopsis organ asymmetry via modulation of KANADI activity. Plant Cell 2005, 17:2899-2910.

33. Baker SC, Robinson-Beers K, Villanueva JM, Gaiser JC, Gasser CS: Interactions among genes regulating ovule development in Arabidopsis thaliana. Genetics 1997, 145:1109-1124

doi:10.1186/1756-0500-4-176

Cite this article as: Krizek: Aintegumenta and Aintegumenta-Like6 regulate auxin-mediated flower development in Arabidopsis. BMC Research Notes 2011 4:176.

\section{Submit your next manuscript to BioMed Central and take full advantage of:}

- Convenient online submission

- Thorough peer review

- No space constraints or color figure charges

- Immediate publication on acceptance

- Inclusion in PubMed, CAS, Scopus and Google Scholar

- Research which is freely available for redistribution 